\title{
A method for computing the three-dimensional radial distribution function of cloud particles from holographic images
}

\author{
Michael L. Larsen ${ }^{1,2}$ and Raymond A. Shaw ${ }^{2}$ \\ ${ }^{1}$ Department of Physics and Astronomy, College of Charleston, Charleston, SC, USA \\ ${ }^{2}$ Department of Physics, Michigan Technological University, Houghton, MI, USA
}

Correspondence: Michael L. Larsen (larsenML@cofc.edu)

Received: 22 February 2018 - Discussion started: 18 April 2018

Revised: 21 June 2018 - Accepted: 2 July 2018 - Published: 19 July 2018

\begin{abstract}
Reliable measurements of the three-dimensional radial distribution function for cloud droplets are desired to help characterize microphysical processes that depend on local drop environment. Existing numerical techniques to estimate this three-dimensional radial distribution function are not well suited to in situ or laboratory data gathered from a finite experimental domain. This paper introduces and tests a new method designed to reliably estimate the three-dimensional radial distribution function in contexts in which (i) physical considerations prohibit the use of periodic boundary conditions and (ii) particle positions are measured inside a convex volume that may have a large aspect ratio. The method is then utilized to measure the three-dimensional radial distribution function from laboratory data taken in a cloud chamber from the Holographic Detector for Clouds (HOLODEC).
\end{abstract}

\section{Introduction}

Cloud droplet clustering is relevant to physical processes like condensational growth (e.g., Srivastava, 1989; Kostinski, 2009), growth by collision-coalescence (e.g., Xue et al., 2008; Onishi et al., 2015), and radiative transfer through clouds (e.g., Kostinski, 2001; Frankel et al., 2017). Consequently, the magnitude of cloud droplet clustering in situ and in the laboratory has been a subject of intense interest for the last 25 years (see, e.g., Baker, 1992; Baumgardner et al., 1993; Brenguier, 1993; Borrmann et al., 1993; Shaw et al., 1998; Uhlig et al., 1998; Davis et al., 1999; Kostinski and Jameson, 2000; Chaumat and Brenguier, 2001; Kostinski and Shaw, 2001; Pinsky and Khain, 2001; Shaw et al., 2002;
Shaw, 2003; Marshak et al., 2005; Larsen, 2006; Lehmann et al., 2007; Salazar et al., 2008; Saw et al., 2008; Small and Chuang, 2008; Baker and Lawson, 2010; Siebert et al., 2010; Bateson and Aliseda, 2012; Larsen, 2012; Saw et al., 2012b; Beals et al., 2015; Siebert et al., 2015; O’Shea et al., 2016).

Most of the in situ studies cited above have utilized airplane-mounted cloud probes that report cloud particle positions in a long, thin, pencil-beam-like volume. For example, the sample volume of the forward scattering spectrometer probe has a cross section of about $0.13 \mathrm{~mm}^{2}$ (Chaumat and Brenguier, 2001). These very thin sample volumes have required the majority of the above investigators to treat cloud particle detections as one-dimensional transects through a three-dimensional medium and appeal to isotropy and spatial homogeneity to infer three-dimensional statistical properties (see, e.g., Holtzer and Collins, 2002). Unfortunately, recent work (Larsen et al., 2014) reveals that - even under isotropic and homogeneous conditions - sampling requirements require far more data than initially suspected to reliably recreate three-dimensional statistics from one-dimensional transects through a cloud.

The most direct and assumption-free way to detect cloud particle clustering is with an instrument that is capable of recording precise particle locations in all three spatial dimensions. This can be carried out with a holographic image of a cloud volume. Some previous holographic studies that explicitly examined three-dimensional cloud particle spatial distributions have been published (see, e.g., Conway et al., 1982; Kozikowsa et al., 1984; Brown, 1989; Borrmann et al., 1993; Uhlig et al., 1998). These pioneering studies were often based on ground-based measurements, included just a few holographic images, and resulted in somewhat conflict- 
ing findings. In most cases, the investigators in the above studies argued that holographic imaging looks like a solid approach to quantify cloud droplet clustering, but the excessive labor required to reconstruct the particle positions from a holographic image made the use of holographic instruments impractical for a large-scale study at the time.

Fortunately, both computational and measurement hardware capabilities, as well as analysis methods, have improved immensely over the last decade, finally bringing holography to a fully digital state that allows for data collection and processing over entire field projects (e.g., Fugal and Shaw, 2009; Beals et al., 2015; O'Shea et al., 2016; Glienke et al., 2017; Schlenczek et al., 2017). For example, the ability to analyze three-dimensional clustering in digital holograms has already been used to identify and eliminate particle shattering effects (e.g Fugal and Shaw, 2009; Jackson et al., 2014; O'Shea et al., 2016) or to identify regions of strong entrainment and inhomogeneous mixing (Beals et al., 2015). These new holographic instruments should also allow for direct characterization of cloud droplet clustering in three dimensions while obtaining sufficient data to yield unambiguous results.

There are many different mathematical tools utilized to characterize the droplet clustering among cloud droplets, each with their own strengths and weaknesses (see, e.g., Baker, 1992; Kostinski and Jameson, 2000; Shaw et al., 2002; Shaw, 2003; Marshak et al., 2005; Baker and Lawson, 2010; Larsen, 2012; Monchaux et al., 2012). Although arguments can be made for any number of these tools, this study focuses on the radial distribution function ( $\operatorname{rdf}$ or $g(r)$ ) because (i) it is a direct scale-localized measure of deviation from perfect spatial randomness, (ii) it is directly related to variances and means through the correlation-fluctuation theorem, (iii) many numerical and theoretical discussions about particle clustering are explicitly presented in terms of the radial distribution function (see, e.g., Balkovsky et al., 2001; Holtzer and Collins, 2002; Collins and Keswani, 2004; Chun et al., 2005; Salazar et al., 2008; Saw et al., 2008; Zaichik and Alipchenkov, 2009; Monchaux et al., 2012; Saw et al., 2012a; Larsen et al., 2014), and (iv) most other common methods of characterizing cloud droplet clustering can be derived from or quantitatively related to a measurement of the radial distribution function (Landau and Lifshitz, 1980; Kostinski and Jameson, 2000; Shaw et al., 2002; Larsen, 2006, 2012).

Although calculation of the three-dimensional radial distribution function from experimentally measured particle position data should be possible, properly accounting for the effects of the edges of the measurement volume can be tricky (Ripley, 1982). (This is in contrast to the much more straightforward calculation of the radial distribution function in numerical simulation domains with periodic boundary conditions, e.g., Reade and Collins, 2000; Wang et al., 2000.) The most commonly utilized method does not make optimal use of the available data and is unable to estimate the radial distribution function at spatial scales larger than approximately one-half the smallest length scale defining the measurement volume $L$. The new method developed in this paper removes both of these limitations.

The remainder of this paper (i) reintroduces the radial distribution function, (ii) presents the methods typically used to estimate the radial distribution function in different experimental and numerical contexts, (iii) outlines the challenges in utilizing these existing methods for experimental data from modern digital holographic images, (iv) presents and tests a new numerical method to calculate the radial distribution function under realistic experimental conditions, and (v) applies this method to real data taken by a digital holographic instrument in a cloud chamber.

\section{Introduction to the radial distribution function}

The radial distribution function is one of the most widely used approaches for characterizing particle clustering in turbulent flows (Monchaux et al., 2012), and is also currently widely used in a variety of other fields including stochastic geometry (e.g., Stoyan et al., 1995), astrophysics (e.g., Martinez and Saar, 2001), granular media (e.g., Lee and Seong, 2016), crystallography (e.g., Cherkas and Cherkas, 2016), and plasma physics (e.g., Erimbetova et al., 2013). The ideas behind its use go back at least a century (e.g., Ornstein and Zernike, 1914), and its wide use permits a large number of different conceptual and notational conventions.

Here, we draw on the introduction given in Landau and Lifshitz (1980), which introduces a similar quantity (the pair correlation function) in terms of the spatial correlation of density fluctuations (Sect. 116 in Landau and Lifshitz (1980)). Let two small disjoint volumes $\mathrm{d} V_{1}$ and $\mathrm{d} V_{2}$ be separated in a statistically homogeneous domain in which the mean number density of particles is given by $\bar{n}=N / V$. The volumes are small enough that detection of more than one particle in $\mathrm{d} V$ is vanishingly small. If the spatial separation between the centers of $\mathrm{d} V_{1}$ and $\mathrm{d} V_{2}$ is $r$, then the probability that both volumes contain a particle can be written as

$p_{(1,2)}(r)=\left(\bar{n} \mathrm{~d} V_{1}\right)\left(\bar{n} \mathrm{~d} V_{2}\right) g(r)$,

where $g(r)$ is the radial distribution function. For perfectly random media with no spatial correlations, $p_{(1,2)}(r)=$ $(\bar{n})^{2} \mathrm{~d} V_{1} \mathrm{~d} V_{2}$ and thus $g(r)=1 \forall r$. If mutual detection in $\mathrm{d} V_{1}$ and $\mathrm{d} V_{2}$ is impossible at separation $r_{\circ}$ (due to, say, excluded volume effects) then $g\left(r_{\circ}\right)=0$. If $g(r)$ exceeds unity, this indicates that there is an enhanced probability of particle separation at scale $r$.

\section{Computing the radial distribution function}

In contexts in which the spatial coordinates of each member of a population of particles are resolved, the radial distribu- 
tion function at scale $r_{\circ}$ can be computed via calculation of

$g(r)=$

observed number of particle pair centers separated by $\left(r_{0}-\delta r<r<r_{0}+\delta r\right)$ number of expected particle pair centers separated by $\left(r_{0}-\delta r<r<r_{\circ}+\delta r\right)$ in a Poisson distribution

where the Poisson distribution has the same total number of particles and volume as the observed system. This can be rewritten algorithmically in any number of dimensions (Saw et al., 2012a) as

$g(r)=\sum_{i=1}^{N} \frac{\psi_{i}(r) / N}{(N-1)\left(\frac{\mathrm{d} V_{r}}{V}\right)}$,

where $\psi_{i}(r)$ is a count of the number of particles having their centers a distance between $r-\delta r$ and $r+\delta r$ from the center of the $i$ th particle in the measurement volume, $N$ is the total number of particles in the measurement volume, $V$ is the measurement volume, and $\mathrm{d} V_{r}$ is the volume of the generalized $n$-dimensional shell between radii $r-\delta r$ and $r+\delta r$.

\subsection{Computing the radial distribution function in one dimension}

Calculation of $g(r)$ (or its related quantity, the paircorrelation function $\eta(r) \equiv g(r)-1)$ has been frequently performed on in situ cloud particle data. Typically, a time series of particle detections is converted to spatial positions along a line utilizing the Taylor frozen-field hypothesis (Saw et al., 2012b). Then, Eq. (3) is modified to

$g_{1-\mathrm{D}}\left(r_{\circ}\right)=\frac{N_{p}\left(r_{\circ}\right)}{\left[N_{\mathrm{in}}\left(r_{\mathrm{o}}\right)+\frac{1}{2} N_{\mathrm{ex}}\left(r_{\mathrm{o}}\right)\right] 2(\delta r)(N-1) / L}$,

where detected particle centers are located between 0 and $L, N_{p}\left(r_{\circ}\right)$ is the number of observed particle centers separated by $r-\delta r<r_{\circ}<r+\delta r, N_{\text {in }}\left(r_{\circ}\right)$ is the number of observed particles detected between $r_{\mathrm{o}}$ and $L-r_{\mathrm{o}}$, and $N_{\mathrm{ex}}\left(r_{\mathrm{o}}\right)$ is the number of observed particles detected between 0 and $r_{\circ}$ plus the number of observed particles detected between $L-r_{\circ}$ and $L$. The factor of $1 / 2$ multiplied by $N_{\text {ex }}\left(r_{\circ}\right)$ is sufficient to account for the edges of the sample volume in the one-dimensional case. Since typically $r_{\circ} \ll L$, this is often simplified to

$g_{1-\mathrm{D}}\left(r_{\circ}\right) \approx \frac{N_{p}\left(r_{\circ}\right)}{2 N(N-1)(\delta r) / L}$.

The above formula has been used in most previous experimental studies computing the radial distribution functions for cloud droplets. In principle, this result can then be used to estimate the three-dimensional radial distribution function following the method outlined in Holtzer and Collins (2002), though the assumptions of statistical homogeneity over the tens to hundreds of kilometers required for obtaining a statistically significant result may be questionable (Larsen et al., 2014). (a)

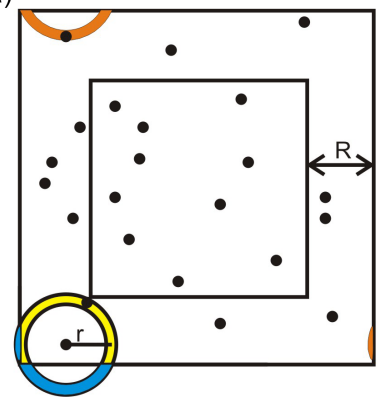

(b)

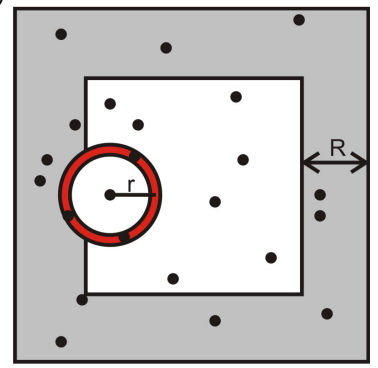

Figure 1. A two-dimensional cartoon of the different ways of traditionally dealing with domain edges when computing the radial distribution function. Panel (a) shows approaches related to a periodic boundary condition approach, whereas (b) illustrates a guard area approach.

\subsection{Computing the radial distribution function in multiple dimensions with periodic boundary conditions}

The three-dimensional radial distribution function can be explicitly computed for cloud droplets in drop-resolving direct numerical simulations. In this context, $g(r)$ can be directly evaluated from Eq. (3) without any modification. The factor that allows computation of the radial distribution function in these scenarios is that the numerical simulations utilize periodic boundary conditions - which extend to the computation of the radial distribution function itself.

When searching for another cloud droplet separated by scale $r_{\circ}-\delta r<r<r_{\circ}+\delta r$, any part of the "search domain" outside of the simulation volume can be wrapped back around through the other side of the computational domain. Since the underlying simulation typically applies this same wrapping boundary condition to resolve particle-fluid and particle-particle interactions, it is consistent with the physics of the simulation to search for particle pairs across the boundaries as well.

A cartoon of this process (shown in two dimensions) can be viewed in Fig. 1a. Some of the circular shell surrounding the particle in the lower left leaves the measurement volume (blue portion). When the data come from a direct numerical simulation, there are no issues in wrapping this volume around to the upper left and lower right corners (to the orange regions), in this case finding an additional particle pair in the upper left. In an actual experiment, however, it is a mistake to argue that the particle in the lower left is correlated to the particle in the upper left at a length scale of $r$ since they are in fundamentally different parts of the flow (i.e., any correlation that does exist is for length scale equal to the nonperiodic distance among the particles usually much greater than $r$ ). 


\subsection{Computing the radial distribution function in multiple dimensions without periodic boundary conditions}

Unfortunately, the technique described in Fig. 1a is not appropriate for most experimental contexts; detected particles on opposite sides of the sample volume do not "know" about each other in the same way that simulations applying periodic boundary conditions do.

The simplest possible solution, albeit the most drastic, in trying to estimate the radial distribution function for finite experimental volumes is to ignore these edge effects entirely. For the cartoon in Fig. 1a, this would be to merely count the one particle detected in the yellow ring and do nothing to account for the blue area at all. Unfortunately, this will cause a computational estimate of $g(r)$ to artificially deviate from unity; actual cloud droplets may exist in the blue area and need to be counted in order to prevent artificial underestimation of $\psi_{i}(r)$ and therefore underestimation of $g(r)$.

Much like in the one-dimensional case, the effects of the edges sometimes can be small enough to make this a minor concern. When the scale of interest $r_{\mathrm{o}}$ is much less than the smallest dimension of the sample volume $(L)$, relatively few particles inside the sample volume will have their $n$ dimensional spherical shells exit the interior of the measurement volume. Unfortunately, however, (i) experimental conditions for cloud droplets will require estimation of $g\left(r_{\circ}\right)$ for $r_{\circ}$ approaching $L$ in order to maximize the evaluated range of $r$, and (ii) the problem becomes more prevalent in higher dimensions and in larger aspect ratios since a larger fraction of the measurement volume is found close to the boundaries.

As noted earlier, this is a problem that has received attention for at least 35 years (Ripley, 1982). Perhaps the most common way to deal with these finite-volume effects is described as "minus sampling" on p. 133 of Stoyan et al. (1995) and illustrated in Fig. 1b. Briefly, one defines a "guard area" within but along the outermost edges of the sampling volume. Particles inside this guard area are not considered part of the actual sample volume $V$, but are used to find pairs for particles within the central (non-guard) part of the measurement volume. For example, the particle in the center of the red circle in Fig. 1b would count three particles between $r-\delta r$ and $r+\delta r$, despite having only two particle pairs within the white region.

Note that $R$ can be either fixed or change with the scale of interest (set $R=r_{\circ}$ when computing $g\left(r_{\circ}\right)$.). The guard area approach does give an unbiased estimator for $g(r)$, but makes sub-optimal use of the data. Two particles within the sample volume could be separated by scale $r_{\circ}-\delta r<r<r_{\circ}+\delta r$ but end up not contributing to the observation, due to the fact that both particles would be in the guard area. Many of the data are lost when using such approaches.

Figure 2 shows another cartoon that demonstrates how limiting the guard area approach can be in different contexts. Here, $R=r$ is only slightly smaller than $L / 2$. The "inner"

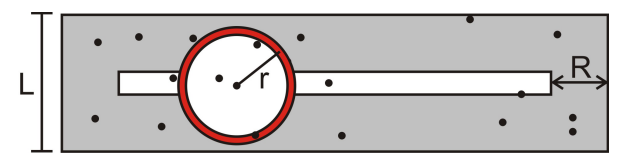

Figure 2. Another cartoon of the guard area technique used to estimate the radial distribution function. Note that the fraction of the particles contributing to the sum in Eq. (3) decreases as the aspect ratio increases, and no estimate of the radial distribution function can be made for any distance larger than half the shortest dimension of the sample volume (when $r \geq L / 2$, no "inner" region remains).

particles that contribute to the sum in Eq. (3) are only the five particles shown inside the central white rectangle. This problem is even worse in $3-\mathrm{D}$, and the aspect ratio shown here is not unrealistic.

The guard area approach is a valid approach for finitevolume cloud measurements, but it imposes a trade-off: either most of the volume can be used, but with severely limited maximum $r$, or the available sample volume is severely reduced in order to accommodate a maximum $r$ that is of the same order as the sample volume linear dimensions. Typically as large a range of $r$ as possible is desired (e.g., in order to have enough scale range to reliably identify power-law exponents), but the associated reduction in available sample volume makes the method quite susceptible to sampling fluctuations. In realistic scenarios in which the entire measurement volume contains only a few hundred to a few thousand particles, sampling considerations make use of the guard area technique prohibitively limiting.

Here, we introduce an alternative edge-correction strategy inspired by Ripley $(1976,1977)$ that we call the "effective volume" radial distribution function method. This approach does not rely on the use of a guard area and allows all retained particles to contribute to the computation of the radial distribution function. We start from a refined expression for the radial distribution function for length scale $r_{j}$ :

$g\left(r_{j}\right)=\sum_{i=1}^{N} \frac{\psi_{i}\left(r_{j}\right) / N}{(N-1)\left(\frac{\mathrm{d} V_{r_{i, j}}}{V}\right)}$.

This is very similar to Eq. (3), except we have made the computationally motivated step of discretizing the set of distances $r_{j}$ and defined a quantity $\mathrm{d} V_{r_{i, j}}$, which is defined as the portion of the volume with a radius between $r_{j}-(\delta r)_{j}$ and $r_{j}+(\delta r)_{j}$ centered on the $i$ th particle that resides within the measurement volume $V$. (For example, in Fig. 1a, $\mathrm{d} V_{r_{i, j}}$ for the highlighted particle would be calculated as that area corresponding to the yellow region.) This depends not only on $r_{j}$ and $(\delta r)_{j}$ but also on the position of the $i$ th particle. Thus, within this method, the denominator is not a constant and must be explicitly calculated particle by particle.

The challenging part of the method is to find $\mathrm{d} V_{r_{i, j}}$; all other parts of the numerical method are the same as have 
been used elsewhere. Although potentially inelegant, $\mathrm{d} V_{r_{i, j}}$ can be found for a wide variety of measurement geometries by generating a measurement geometry-dependent look-up table. This can be accomplished by computing values of $\mathrm{d} V_{r_{i, j}}$ in a dense grid of possible positions of each detected particle and at each desired distance $r_{j}$. Since for convex volumes it is empirically found that $\mathrm{d} V_{r_{i, j}}$ is relatively smooth over the measurement domain, one can then assign $\mathrm{d} V_{r_{i, j}}$ for the $i$ th particle at the $j$ th radial distance by utilizing the lookup-table-stored grid point closest to the actual particle position.

There are multiple ways to generate the proposed look-up table. In this study, we have populated the interior of the measurement volume with a regular dense grid with grid spacing $s$. Then, for each grid point and for each scale of interest $r_{j}$, the number of other grid points contained in a shell with inner and outer radii $r_{j}-(\delta r)_{j}$ and $r_{j}+(\delta r)_{j}$ are counted. This is then compared to the number of grid points that would be contained in a shell of the same volume within an infinite grid with the same grid spacing $s$. The ratio of these two counts is then multiplied by the true volume of the shell $\mathrm{d} V_{j}$ to give $\mathrm{d} V_{r_{i, j}}$. This method allows for reliable estimation of $\mathrm{d} V_{r_{i, j}}$ without having to mathematically calculate the quantity analytically, which would require rather lengthy treatments of possible boundary-shell intersection geometries (especially in three dimensions).

Conceptually, the algorithm uses the $i$ th term in the sum in Eq. (6) to find an appropriately weighted contribution to $g(r)$ from the $i$ th particle; when summed over all particles in the measurement volume, the expression gives an estimator for $g(r)$ that accounts for edge effects. This weighting factor appears in the denominator and is based on a term that depends on how close the $i$ th particle is to the edge of the measurement volume; if the particle in question is within $r_{j}$ of the edge of the measurement volume, only the portion of the $n$-dimensional spherical shell $\mathrm{d} V$ that still lies within the measurement domain is used.

The effective volume method allows for any investigatorchosen values of $r_{j}$ and $(\delta r)_{j}$, allows for as fine of a tessellation of the measurement volume as desired for precision in the look-up table, and can be used even for $r_{j}>L$. Once the look-up table is generated it can be applied to all data in a data set, assuming the instrument measurement volume shape and size are constant. It should be further noted that symmetry in the measurement volume shape can be used to reduce the number of normalization volumes that need to be calculated. For example, in a rectangular parallelepiped only one octant (corner) of the measurement volume needs to be in the look-up table. More explicit detail on how to implement this method is presented in the appendix.

\section{Testing the effective volume method}

The effective volume method described above was implemented for two different geometries - a cubical geometry (to allow for useful comparisons to the well-known and frequently utilized guard area technique) as well as for an applied geometry to match a real instrument. For each geometry, we present two tests: a homogeneous Poisson distribution and a Matérn cluster process.

A homogeneous Poisson distribution is the gold standard of spatial randomness. Within a homogeneous Poisson distribution, all particles are placed independently with a spatial density function uniform over the measurement domain. By construction, $g(r)=1 \forall r$ within a volume with particles distributed according to a homogeneous Poisson distribution.

A Matèrn cluster process (see, e.g., Stoyan et al., 1995; Martinez and Saar, 2001; Schabenberger and Goway, 2005; Larsen, 2012) is commonly used in stochastic geometry because it is (i) statistically homogeneous, (ii) easy to simulate in any number of spatial dimensions, and (iii) has a known closed-form expression for its radial distribution function.

\subsection{Simulations in a cube}

Both distributions described above were simulated within a unit cube with both guard area and effective volume computation methods.

For the guard area computation method, a fixed $R=0.1$ was used around the outside edges of the cube. This should allow for unbiased estimation for $g(r)$ when $r<0.1$, but it is expected that $g(r>0.1)$ will underestimate the true values.

The effective volume computation method was computed by creating a look-up table for a cubical volume. Due to the symmetry of the volume, only one octant of the cube had to be included in the look-up table. To minimize the size of the look-up table required, the density of the tessellation of the cubical measurement volume was varied depending on the distance to the boundary - with points near the boundary having the densest collection of look-up table entries.

A total of 100 simulations of each volume were averaged together and the results are displayed in Fig. 3. Except for the smallest scales (for which sampling variability is still nonnegligible, even after a total of 100 simulations), agreement between the effective volume method and the theoretical $g(r)$ curves expected is excellent and comparable to the values observed for the more commonly used guard area approach.

In this case, the guard area approach involves summing over less than half (on average $48.8 \%$ ) of particles in the measurement volume, which can help to explain the larger scatter of observed $g(r)$ for small $r$ using this approach. Note also the deviation from the theoretical $g(r)$ curve in both tests for the guard area approach for $r>0.1$, consistent with pushing the approach beyond its domain of applicability. 


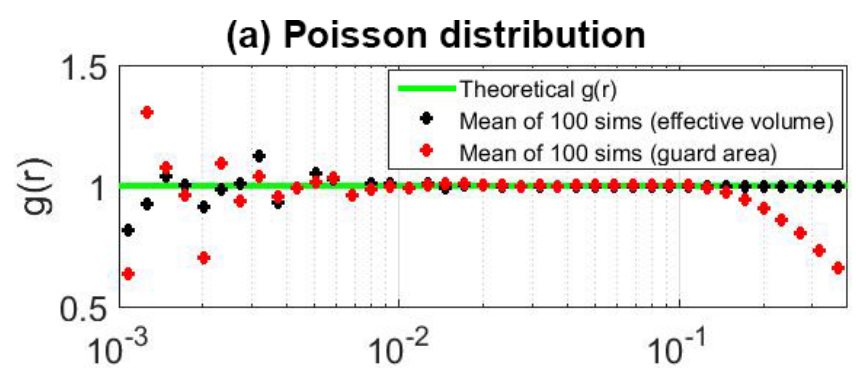

(b) Matérn cluster process

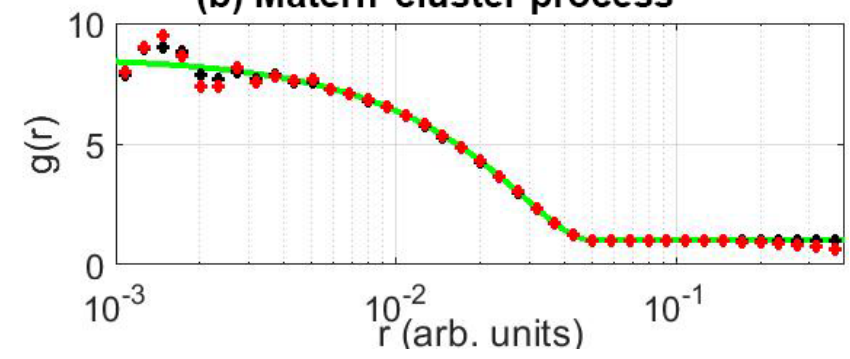

Figure 3. First verification of the method for calculating $g(r)$ described in the main text. Here, theoretical curves of $g(r)$ are compared to both the effective volume method and the guard area method (using a fixed guard area of 0.1 times the side length of the cube). In the top panel, 100 different 10000 particle Poisson distributions were created. Deviations from $g(r)=1$ in both methods are observed at small $r$ values due to sampling fluctuations. Panel (b) shows similar results from 100 simulations of a Matérn cluster process (with a mean of 10000 total particles and a cluster length of 0.025$)$. Note that in both panels the guard area approach begins to fail as expected for $r>0.1$.

\subsection{Case study: the Holographic Detector for Clouds (HOLODEC)}

Although the effective volume approach introduced here performs approximately as well as the more traditional guard area approach in cubical volumes, the development of the new method was primarily motivated by a desire to estimate the radial distribution function in contexts in which the guard area approach will not work. As noted previously, when estimates of $g(r)$ are desired for $r \gtrsim L / 2$ and/or the aspect ratio of the measurement volume deviates substantially from unity, the guard area approach becomes ineffective.

An example of an instrument that is subject to these limitations and is relevant for studying cloud particle clustering is the Holographic Detector for Clouds (HOLODEC).

\subsubsection{Introduction to HOLODEC}

HOLODEC is an in-line digital holography instrument explicitly designed to explore cloud microstructure (Fugal et al., 2004; Fugal and Shaw, 2009; Spuler and Fugal, 2011). The instrument has previously been used to examine drop size distribution and liquid water content fluctuations on the centimeter scale (Beals et al., 2015), and the behavior of the

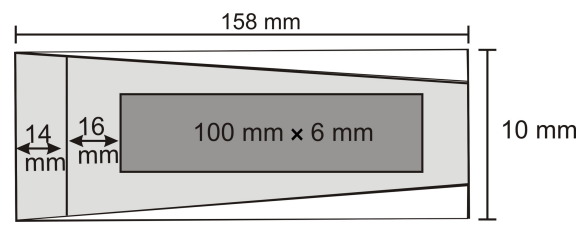

Figure 4. A two-dimensional cartoon of the HOLODEC sample volume (not to scale). The leftmost vertical line in the figure indicates the hologram plane. The light grey region indicates areas of maximum sensor sensitivity (the slope of the angled lines marking the edge of the light grey region has been greatly magnified for aid in visualization). The vertical lines 14 and $158 \mathrm{~mm}$ from the left edge of the figure mark the positions of the optical windows; near these windows there is evidence of artificially generated particles due to instrument-induced particle fragmentation. The volume simulated here corresponds to the darker central grey rectangle (parallelepiped in 3-D), where the instrument retains approximately uniform sensitivity, particle locations and sizes are believed to be accurate, and the number of small particles generated due to fragmentation on the instrument is believed to be negligible.

instrument has been validated by comparison to co-collected cloud droplet probe (CDP) and 2DC optical array probe data in different parts of the particle size domain (Glienke et al., 2017).

A processed HOLODEC hologram reports droplet positions in a volume that is approximately $1 \mathrm{~cm} \times 1 \mathrm{~cm} \times 15.8 \mathrm{~cm}$ with sensitivity to all droplets with sizes greater than about $6.5 \mu \mathrm{m}$. The positional uncertainty for each drop is approximately $10 \mu \mathrm{m}$ along the short sides of the sample volume and about $100 \mu \mathrm{m}$ along the longer side (Yang et al., 2005).

A two-dimensional cartoon of the HOLODEC sample volume is shown in Fig. 4. Although particles out to $158 \mathrm{~mm}$ (or further) from the hologram plane are potentially visible, the optical windows 14 and $158 \mathrm{~mm}$ from the hologram plane limit the air-exposed field of view to the approximately $14 \mathrm{~cm}$ distance between the windows. Additionally, the spatial domain of instrumental sensitivity is not a perfect parallelepiped. Preliminary analyses of data suggest that there may be some decreased sensitivity near the edges of the sample volume, and - when mounted on an aircraft - drops can be created by fragmentation near the optical windows (Fugal and Shaw, 2009). Consequently, to ensure data fidelity when used with real data, a conservative sub-volume of each hologram is selected as the measurement volume for analysis. This sub-volume was selected to be in the central part of each hologram where the data are expected to be most reliable. Thus, the used HOLODEC sample volume is a $6 \mathrm{~mm} \times 6 \mathrm{~mm} \times 10 \mathrm{~cm}$ rectangular parallelepiped.

\subsubsection{Simulations within the instrument domain}

The same two tests (homogeneous Poisson distribution and Matérn cluster process) were simulated within the paral- 
(a) Poisson distribution

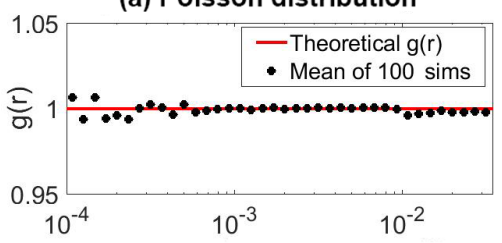

(b) Matérn cluster process

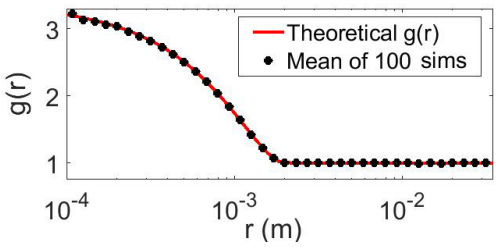

Figure 5. Verification that the effective volume method for calculating $g(r)$ as described in the main text works for non-cubical sample volumes with realistic aspect ratios. In panel (a), 100 different simulations of a Poisson distribution (perfect spatial randomness) were created by placing 10000 particles within a sample volume with the same dimensions as the HOLODEC sample volume. The mean of the 100 simulations agrees very well with the theoretical $g(r)=1$ curve. An unrealistically large number of particles were used for each simulation in order to minimize the sampling concerns. Note that though the agreement is very good, the most pronounced deviations from the theoretical curve still occur as expected at small spatial scales. In panel (b), 100 different simulations of a Matérn cluster process were generated and compared to the known theoretical expression (see, e.g., Larsen et al., 2014). Clearly, agreement between the mean of the simulations and the theoretical curve is excellent.

lelepiped sample volume of the HOLODEC. A new lookup table for this geometry was generated and used to calculate $g(r)$ for each of 100 different simulations, with the mean value of $g(r)$ compared to theoretical expectations and shown in Fig. 5. The guard area method for calculating $g(r)$ is not shown since it is susceptible to substantial sampling variability at all scales and cannot be used at all for any scale larger than $3 \mathrm{~mm}$ (the entire volume is then the guard area).

In general, the agreement between the theoretical expressions for $g(r)$ and the measured $g(r)$ is excellent, and suggests that the effective volume radial distribution function computational method should work for real data.

\subsubsection{Real data}

To test the claim made above, a proof-of-principle analysis was completed using real HOLODEC data acquired inside a laboratory cloud chamber driven by Rayleigh-Bénard convection (Chandrakar et al., 2016, 2017; Chang et al., 2016; Desai et al., 2018).

The radial distribution functions of the eight holograms with the largest numbers of detected drops are shown in Fig. 6. For these eight holograms, there were an average of about 185 cloud drops per cubic centimeter within the mea-

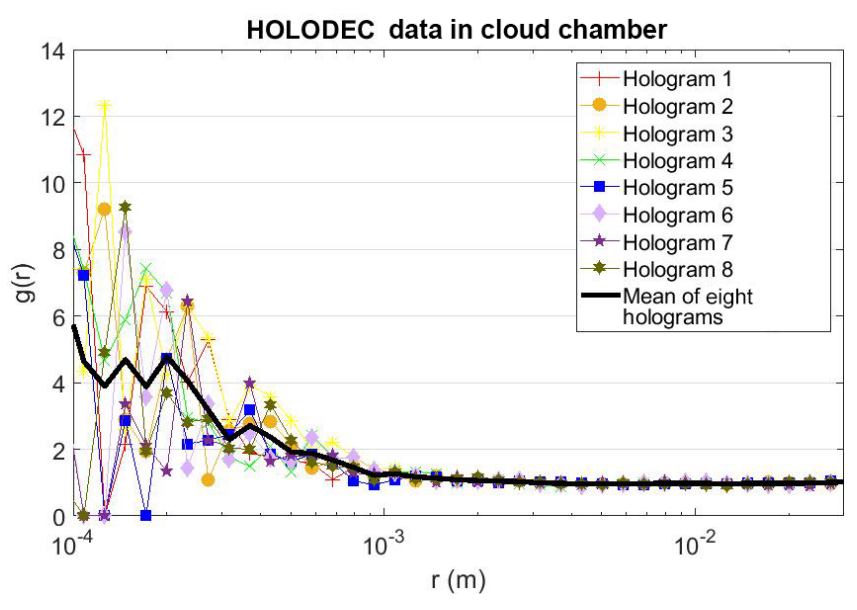

Figure 6. The measured radial distribution functions for eight different holograms and their mean for HOLODEC data taken in the cloud chamber. Clearly sampling variability is still pronounced at small spatial scales, but some evidence of scale-dependent clustering seems possible.

surement domain (which is reasonable compared to naturally occurring clouds).

These single-hologram results are noisy due to the sampling uncertainty (especially for the smallest spatial scales), but it is clear that there is some evidence of scale-dependent clustering revealed for $r \lesssim 1 \mathrm{~mm}$. Quantitative comparisons to theoretical expectation are too much to ask for from such a limited data set, but it is promising to note that the $g(r)$ curve decays as expected to unity for scales larger than a few $\eta$ (where $\eta$ is the turbulent Kolmogorov scale - nominally $1 \mathrm{~mm}$ for the atmosphere). Additionally, the observed increase in $g(r)$ with decreasing length scale $r$ within the dissipative range is consistent with expectations for inertial clustering of particles in a turbulent flow (Reade and Collins, 2000; Ayala et al., 2008; Saw et al., 2012a). Despite these encouraging features, it is important to note that here we merely present Fig. 6 to demonstrate that the algorithm gives plausible results for real data; quantitative analysis of these chamber data with so few holograms would be premature.

Actually measuring the three-dimensional radial distribution function for in situ flight data will still be challenging, even with the aid of the algorithm introduced here. The radial distribution function curves shown in Fig. 6 suggest that individual holograms likely do not give statistically reliable information on spatial scales of microphysical interest; thus some means of combining information from multiple holograms will need to be explored in the future. The number of holograms needed for extraction of in situ radial distribution function values will critically depend on data and instrument parameters including (i) the spatial scale of interest $(r)$, (ii) the spatial resolution of interest $(\mathrm{d} r)$, (iii) the size and shape of the measurement volume of the instrument, (iv) the density of cloud particles, and (v) the acceptable level of un- 
certainty in the extracted radial distribution function. For example, a crude estimate with fixed $\mathrm{d} r=100 \mu \mathrm{m}$ for the data presented here suggests that estimation to within $1 \%$ uncertainty in $g(r)$ requires only one hologram for reliable estimation of $g(r=1 \mathrm{~cm})$ but approximately 60 holograms for reliable estimation of $g(r=1 \mathrm{~mm})$ and almost 3000 holograms for reliable estimation of $g(r=100 \mu \mathrm{m})$. However, the number of required holograms may be more modest depending on the drop number concentration, usable measurement volume of the sensor, scale of physical interest, spatial resolution of $g(r)$ required, and/or the level of acceptable uncertainty in the estimate of $g(r)$. Current work with in situ data has revealed promising results, but here our emphasis has been on proving the viability of the numerical algorithm.

\section{Conclusions}

Understanding the effects of cloud particle clustering on microphysical processes requires reliable estimation of the three-dimensional radial distribution function. Previous studies have obtained this information by utilizing onedimensional measurements of cloud particle positions to infer scale-dependent clustering, but these methods have been shown to carry large uncertainties. In the hope of finding an alternative way of characterizing cloud particle clustering without such restrictive underlying assumptions and/or uncertainties, measurement of the radial distribution function for in situ data in three dimensions is desired.
Comparing measurements with theory and numerical simulation relies on estimating $g(r)$ over a wide range of spatial scales and making optimal use of the measured data to combat sampling uncertainties. Because the aspect ratios of the holographic instruments designed to explore threedimensional cloud microstructure are large and the radial distribution function must be estimated on scales exceeding the smallest dimension of the measurement volume, standard computational methods that use spatial information to estimate the radial distribution function are not adequate.

Here, a new method was introduced that explicitly considers each particle's position within the measurement volume in the radial distribution function computation. This method allows for calculating the radial distribution function for scales larger than the shortest physical dimension of the measurement volume and makes more optimal use of the measured data. This effective volume method was tested in two different geometries, compared to standard computational methods with simulated data in a unit cube, and validated in a more realistic sampling scenario.

Preliminary results confirm that use of the effective volume method should enable the use of airborne digital holography data to compute in situ three-dimensional radial distribution functions for cloud droplets.

Data availability. The HOLODEC data associated with the analysis in Sect. 4.2.3 are available from the authors by request. 


\section{Appendix A: Basic structure of codes to use the effective volume method}

The effective volume method to calculate the radial distribution function relies on two codes - one to generate a lookup table for the measurement volume, and another to use the look-up table and data to compute the radial distribution function. This appendix outlines the basic structure utilized for each of these codes.

\section{A1 Generating the look-up table}

Required inputs from the user include the following: physical domain of sample volume, set of radii $r_{j}$ and associated ranges $(\delta r)_{j}$, and grid tessellation scale $s$ (as small as computationally feasible).

1. Tessellate the interior of the sample volume domain at scale $s$, giving a total of $M$ grid points.

2. For each radius $r_{j}$, and for each grid point $i=1$ : $M$, compute the number of grid points inside the $n$ dimensional shell centered on the $i$ th grid point with inner and outer radii $r_{j}-(\delta r)_{j}$ and $r_{j}+(\delta r)_{j}$, respectively. Store the result as $a(i, j)$.

3. Tessellate an $n$-dimensional cube at scale $s$ with side lengths $2\left[\max \left(r_{j}+\left(\delta r_{j}\right)\right)\right]$.

4. For each radius $r_{j}$, compute the number of grid points inside the $n$-dimensional shell centered on the center of the $n$-dimensional cube with inner and outer radii $r_{j}-(\delta r)_{j}$ and $r_{j}+(\delta r)_{j}$, respectively. Store the result as $b(j)$.

5. Compute the factor $\operatorname{norm}(i, j)=a(i, j) / b(j)$.

\section{A2 Using the look-up table and data to compute a radial distribution function}

Required inputs include the same set of inputs utilized to generate the look-up table and $N$ different $n$-dimensional particle positions.

1. Load the look-up table.

2. For each radius $r_{j}$, calculate the volume of the $n$ dimensional spherical shell between radii $r_{j}-(\delta r)_{j}$ and $r_{j}+(\delta r)_{j}$. Store the results as $\mathrm{d} V(j)$.

3. For each particle $k=1: N$ and for each radius $r_{j}$

(a) count the number of other particles that are between $r_{j}-(\delta r)_{j}$ and $r_{j}+(\delta r)_{j}$ from the $k$ th particle and store the result as $\psi(k, j)$.

(b) identify the closest entry in the look-up table $i$ to the associated position of the $k$ th particle; store as $p$.

(c) assign $\mathrm{d} V r(k, j)=\mathrm{d} V(j) \cdot \operatorname{norm}(p, j)$.

(d) use $\mathrm{d} \operatorname{Vr}(k, j)$ and $\psi(k, j)$ to compute the $k$ th term of the sum for $g(r)$ following Eq. (6) to give $g(k, j)$.

4. Compute and return $g(j)=\sum_{k=1}^{N} g(k, j)$. 
Competing interests. The authors declare that there is no conflict of interest.

Acknowledgements. This work was supported by the US National Science Foundation through grants AGS-1532977 (MLL) and AGS-1623429 (RAS). Special thanks to Alexander Kostinski, Susanne Glienke, and Neel Desai for helpful discussions and help with accessing and interpreting the HOLODEC data from the $\Pi$ Chamber.

Edited by: Szymon Malinowski

Reviewed by: three anonymous referees

\section{References}

Ayala, O., Rosa, B., Wang, L.-P., and Grabowski, W.: Effects of turbulence on the geometric collision rate of sedimenting droplets. Part I: Results from direct numerical simulation, New J. Phys., 10, 075015, https://doi.org/10.1088/13672630/10/7/075015, 2008.

Baker, B.: Turbulent entrainment and mixing in clouds: A new observational approach, J. Atmos. Sci., 49, 387-404, 1992.

Baker, B. and Lawson, R.: Analysis of tools used to quantify droplet clustering in clouds, J. Atmos. Sci., 67, 3355-3367, 2010.

Balkovsky, E., Falkovich, G., and Fouxon, A.: Intermittent distribution of inertial particles in turbulent flows, Phys. Rev. Lett., 86, 2790-2793, 2001.

Bateson, C. and Aliseda, A.: Wind tunnel measurements of the preferential concentration of inertial droplets in homogenous isotropic turbulence, Exp. Fluids, 52, 1373-1387, 2012.

Baumgardner, D., Baker, B., and Weaver, K.: A technique fo rthe measurements of cloud structure in centimeter scales, J. Atmos. Ocean. Technol., 10, 557-565, 1993.

Beals, M., Fugal, J., Shaw, R., Lu, J., Spuler, S., and Stith, J.: Holographic measurements of inhomogeneous cloud mixing at the centimeter scale, Science, 350, 87-90, 2015.

Borrmann, S., Jaenicke, R., and Neumann, P.: On spatial distributions and inter-droplet distances measured in stratus clouds with in-line holography, Atmos. Res., 29, 229-245, 1993.

Brenguier, J.-L.: Observations of cloud microstructure at the centimeter scale, J. Appl. Meteorol., 32, 783-793, 1993.

Brown, P.: Use of holography for airborne cloud physics measurements, J. Atmos. Ocean. Technol., 6, 293-306, 1989.

Chandrakar, K., Cantrell, W., Chang, K., Ciochetto, D., Niedermeier, D., Ovchinnikov, M., Shaw, R., and Yang, F.: Aerosol indirect effect from turbulence-induced broadening of cloud-droplet size distributions, P. Natl. Acad. Sci., 113, 14243-14248, 2016.

Chandrakar, K., Cantrell, W., Ciochetto, D., Karki, S., Kinney, G., and Shaw, R.: Aerosol removal and cloud collapse accelerated by supersaturation fluctuations in turbulence, Geophys. Res. Lett., 44, 4359-4367, 2017.

Chang, K., Bench, J., Brege, M., Cantrell, W., Chandrakar, K., Ciochetto, D., Mazzoleni, C., Mazzoleni, L., Niedermeier, D., and Shaw, R.: A laboratory facility to study gas-aerosol-cloud interactions in a turbulent enviornment: The $\Pi$ chamber, B. Am. Meteor. Soc., 97, 2343-2358, 2016.
Chaumat, L. and Brenguier, J.: Droplet spectra broadening in cumulus clouds. Part II: Microscale droplet concentration inhomogeneities, J. Atmos. Sci., 58, 642-654, 2001.

Cherkas, N. and Cherkas, S.: Model of the radial distribution function of pores in a layer of porous aluminum oxide, Crystallogr. Rep., 61, 285-290, 2016.

Chun, J., Koch, D., Rani, S., Ahluwalia, A., and Collins, L.: Clustering of aerosol particles in isotropic turbulence, J. Fluid Mech., 536, 219-251, 2005.

Collins, L. and Keswani, A.: Reynolds number scaling of particle clustering in turbulent aerosols, New J. Phys., 6, 1-17, 2004.

Conway, B., Caughey, S., Bentley, A., and Turton, J.: Ground-based and airborne holography of ice and water clouds, Atmospheric Environment, 16, 1193-1207, 1982.

Davis, A., Marshak, A., Gerber, H., and Wiscombe, W.: Horizontal structure of marine boundary layer clouds from centimeter to kilometer scales, J. Geophys. Res., 104, 6123-6144, 1999.

Desai, N., Chandrakar, K., Chang, K., Cantrell, W., and Shaw, R.: Influence of microphysical variability on stochastic condensation in a turbulent laboratory cloud, J. Atmos. Sci., 75, 189-201, 2018.

Erimbetova, L., Davletov, A., Kudyshev, Z. A., and Mukhametkarimov, Y. S.: Influence of polarization phenomena on radial distribution function of dust particles, Contrib. Plasm. Phys., 53, 414418, 2013.

Frankel, A., Iaccarino, G., and Mani, A.: Optical depth in particleladen turbulent flows, J. Quant. Spesc. Ra., 201, 10-16, 2017.

Fugal, J. P. and Shaw, R. A.: Cloud particle size distributions measured with an airborne digital in-line holographic instrument, Atmos. Meas. Tech., 2, 259-271, https://doi.org/10.5194/amt-2259-2009, 2009.

Fugal, J., Shaw, R., Saw, E.-W., and Sergeyev, A.: Airborne digital holographic system for cloud particle measurements, Appl. Optics, 43, 5987-5995, 2004.

Glienke, S., Kostinski, A., Fugal, J., Shaw, R., Borrmann, S., and Stith, J.: Cloud droplets to drizzle: Contribution of transition drops to microphysical and optical properties of marine stratocumulus clouds, Geophys. Res. Lett., 44, 8002-8010, https://doi.org/10.1002/2017GL074430, 2017.

Holtzer, G. and Collins, L.: Relationship between the intrinsic radial distribution function for an isotropic field of particles and lower-dimensional measurements, J. Fluid Mech., 459, 93-102, https://doi.org/10.1017/S0022112002008169, 2002.

Jackson, R., McFarquhar, G., Stith, J., Beals, M., Shaw, R., Jensen, J., Fugal, J., and Korolev, A.: An assessment of the impact of antishattering tips and artifact removal techniques on cloud ice size distributions measured by the $2 \mathrm{D}$ cloud probe, J. Atmos. Ocean. Technol., 31, 2567-2590, 2014.

Kostinski, A.: On the extinction of radiation by a homogeneous but spatially correlated random medium, J. Opt. Soc. Am. A, 18, 1929-1933, https://doi.org/10.1364/JOSAA.18.001929, 2001.

Kostinski, A.: Simple approximations for condensational growth, Environ. Res. Lett., 4, 015005, https://doi.org/10.1088/17489326/4/1/015005, 2009.

Kostinski, A. and Jameson, A.: On the spatial distribution of cloud particles, J. Atmos. Sci., 57, 901-915, 2000.

Kostinski, A. and Shaw, R.: Scale-dependent droplet clustering in turbulent clouds, J. Fluid Mech., 434, 389-398, 2001. 
Kozikowsa, A., Haman, K., and Supronowicz, J.: Preliminary results of an investigation of the spatial distribution of fog droplets by a holographic method, Q. J. Roy. Meteorol. Soc., 110, 65-73, 1984.

Landau, L. and Lifshitz, E.: Statistical Physics, Butterworth Heinemann, Oxford, UK, 1980.

Larsen, M.: Studies of discrete fluctuations in atmospheric phenomena, Ph.D. thesis, Michigan Technological University, 2006.

Larsen, M.: Scale localization of cloud particle clustering statistics, J. Atmos. Sci., 69, 3277-3289, https://doi.org/10.1175/JAS-D12-02.1, 2012.

Larsen, M., Briner, C., and Boehner, P.: On the recovery of 3D spatial statistics of particles from 1D measurements: Implications for airborne instruments, J. Atmos. Ocean. Technol., 31, 20782087, https://doi.org/10.1175/JTECH-D-14-00004.1, 2014.

Lee, K. and Seong, W.: Percus-Yevick radial distribution function calculation for a water-saturated granular medium, Ocean Eng., $116,268-272,2016$.

Lehmann, K., Siebert, H., Wendisch, M., and Shaw, R.: Evidence for inertial droplet clustering in weakly turbulent clouds, Tellus, 59B, 57-65, 2007.

Marshak, A., Knyazikhin, Y., Larsen, M., and Wiscombe, W. J.: Small-scale drop size variability: Empirical models for dropsize-dependent clustering in clouds, J. Atmos. Sci., 62, 551-558, 2005

Martinez, V. and Saar, E.: Statistics of the Galaxy Distribution, CRC Press, Boca Raton, 456 pp., 2001.

Monchaux, R., Bourgoin, M., and Cartellier, A.: Analyzing preferential concentration and clustering of inertial particles in turbulence, Int. J. Multiphas. Flow, 40, 1-18, 2012.

Onishi, R., Matsuda, K., and Takahashi, K.: Lagrangian tracking simulation of droplet growth in turbulence - Turbulence enhancement of autoconversion rate, J. Atmos. Sci., 72, 25912607, 2015.

Ornstein, L. and Zernike, F.: Accidental deviations of density and opalescence at the critical point of a single substance, KNAW Proc., 17, 793-806, 1914.

O'Shea, S., Choularton, T., Lloyd, G., Crosier, J., Bower, K., Gallagher, M., Abel, S., Cotton, R., Brown, P., Fugal, J., Schlenczek, O., Borrmann, S., and Pickering, J.: Airborne observations of the microphysical structure of two contrasting cirrus clouds, J. Geophys. Res.-Atmos., 121, 13510-13536, 2016.

Pinsky, M. and Khain, A.: Fine structure of cloud droplet concentration as seen from the Fast-FSSP measurements. Part I: Method of analysis and preliminary results, J. Appl. Meteorol., 40, 15151537, 2001.

Reade, W. and Collins, L.: Effect of preferential concentration on turbulent collision rates, Phys. Fluids, 12, 2530-2540, 2000.

Ripley, B.: The second-order analysis of stationary point processes, J. Appl. Probab., 13, 255-266, 1976.

Ripley, B.: Modelling spatial paterns (with discussion), J. Roy. Stat. Soc., B39, 172-212, 1977.

Ripley, B.: Edge effects in spatial stochastic processes, in: Statistics in Theory and Practice: Essays in Honour of Bertil Matérn, edited by: Ranneby, B., 242-262, 1982.

Salazar, J., Jong, J. D., Cao, L., Woodward, C., Meng, H., and Collins, L.: Experimental and numerical inverstigation of inertial particle clustering in isotropic turbulence, J. Fluid Mech., 600, 245-256, 2008.
Saw, E.-W., Shaw, R., Ayyalasomayajula, S., Chuang, P., and Gylfason, A.: Inertial particle clustering of particles in highReynolds-number turbulence, Phys. Rev. Lett., 100, 214501, https://doi.org/10.1103/PhysRevLett.100.214501, 2008.

Saw, E.-W., Salazar, J., Collins, L., and Shaw, R.: Spatial clustering of polydisperse inertial particles in turbulence: I. Comparing simulation with theory, New J. Phys., 14, 105030, https://doi.org/10.1088/1367-2630/14/10/105030, 2012a.

Saw, E.-W., Shaw, R., Salazar, J., and Collins, L.: Spatial clustering of polydisperse inertial particles in turbulence: II. Comparing simulation with experiment, New J. Phys., 14, 105031, https://doi.org/10.1088/1367-2630/14/10/105031, 2012b.

Schabenberger, O. and Goway, C.: Statistical Methods for Spatial Data Analysis, Chapman and Hall/CRC, Boca Raton, 504 pp., 2005.

Schlenczek, O., Fugal, J., Lloyd, G., Bower, K., Choularton, T., Flynn, M., Crosier, J., and Borrmann, S.: Microphysical properties of ice crystal precipitation and surface-generated ice crystals in a high alpine environment in Switzerland, J. Appl. Meteorol. Climatol., 56, 433-453, 2017.

Shaw, R.: Particle-turbulence interactions in atmospheric clouds, Annu. Rev. Fluid Mech., 35, 183-227, https://doi.org/10.1146/annurev.fluid.35.101101.161125, 2003.

Shaw, R., Reade, W., Collins, L., and Verlinde, J.: Preferential concentration of cloud droplets by turbulence: Effects on the early evolution of cumulus cloud droplet spectra, J. Atmos. Sci., 55, 1965-1976, 1998.

Shaw, R., Kostinski, A., and Larsen, M.: Towards quantifying droplet clustering in clouds, Q. J. Roy. Meteorol. Soc., 128, 1043-1057, https://doi.org/10.1256/003590002320373193, 2002.

Siebert, H., Gerashchenko, S., Gylfason, A., Lehmann, K., Collins, L., Shaw, R., and Warhaft, Z.: Towards understanding the role of turbulence on droplets in clouds: In situ and laboratory measurements, Atmos. Res., 97, 426-437, 2010.

Siebert, H., Shaw, R. A., Ditas, J., Schmeissner, T., Malinowski, S. P., Bodenschatz, E., and Xu, H.: High-resolution measurement of cloud microphysics and turbulence at a mountaintop station, Atmos. Meas. Tech., 8, 3219-3228, https://doi.org/10.5194/amt8-3219-2015, 2015.

Small, J. and Chuang, P.: New observations of precipitation initiation in warm cumulus clouds, J. Atmos. Sci., 65, 2972-2982, 2008.

Spuler, S. and Fugal, J.: Design of an in-line, digital holographic imaging system for airborne measurement of clouds, Appl. Optics, 50, 1405-1412, 2011.

Srivastava, R.: Growth of cloud drops by condensation: A criticism of currently-accepted theory and a new approach, J. Atmos. Sci., 46, 869-887, 1989.

Stoyan, D., Kendall, W., and Mecke, J.: Stochastic Geometry and its Applications, Wiley, Chichister, England, 436 pp., 1995.

Uhlig, E.-M., Borrmann, S., and Jaenicke, R.: Holographic in-situ measurements of the spatial droplet distribution in stratiform clouds, Tellus, 50B, 377-387, 1998.

Wang, L., Wexler, A., and Zhou, Y.: Statistical mechanical description and modeling of trubulent collision of inertial particles, J. Fluid Mech., 415, 117-153, 2000. 
Xue, Y., Wang, L.-P., and Grabowski, W.: Growth of cloud droplets by turbulent collision-coalescence, J. Atmos. Sci., 65, 331-356, 2008.

Yang, W., Kostinski, A., and Shaw, R.: Depth-of-focus reduction for digital in-line holography of particle fields, Opt. Lett., 30, 13031305, 2005.
Zaichik, L. and Alipchenkov, V.: Statistical models for predicting pair dispersion and particle clustering in isotropic turbulence and their applications, New J. Phys., 11, 103018, https://doi.org/10.1088/1367-2630/11/10/103018, 2009. 